\title{
Superconducting Strand Properties at Each Production Stage of the CMS Solenoid Conductor Manufacturing
}

\author{
B. Blau, D. Campi, B. Curé, M. Greco, F. Kircher, R. Liikamaa, J. Seppälä, R. Smith, and L. Vieillard
}

\begin{abstract}
The Compact Muon Solenoid (CMS) detector is one of the general-purpose particle detectors presently being built for the LHC project at CERN. The superconducting CMS solenoid will produce a magnetic field of $4 \mathrm{~T}$ in a bore $6 \mathrm{~m}$ in diameter and $12.5 \mathrm{~m}$ long. The coil is wound from 20 high purity aluminum-stabilized $\mathrm{NbTi}$ conductors with a total length of $45 \mathrm{~km}$. The main part of the structural integrity of the CMS coil is ensured by aluminum-alloy reinforcement welded to the high purity aluminum stabilizer of the conductor. The Rutherford type superconducting cable within the stabilizer consists of 32 copper-stabilized multifilamentary NbTi strands each with $\mathrm{Nb}$ barrier. The strands are optimized with respect to a high critical current density $\left(>3000 \mathrm{~A} / \mathrm{mm}^{2}\right.$ at $5 \mathrm{~T}$, 4.2 K).

Approximately $1950 \mathrm{~km}$ of superconducting strand has been produced from 148 extrusion billets and cabled in unit lengths of $2.65 \mathrm{~km}$. All strands within a cable can unambiguously be identified by distinctive patterns of the NbTi filaments. The statistics of $I_{c}$ measurements, n-value, copper RRR and $(\mathrm{Cu}+\mathrm{Nb}) / \mathrm{NbTi}$ ratio are presented. Since the strands are subjected to potentially dangerous high temperatures and mechanical strain during the different conductor production steps it was important to trace the critical current properties of the strand as it progressed through the conductor fabrication process. The paper presents results of $I_{c}$ measurements, which were performed on all individual strands extracted from conductor samples taken at each step of the manufacturing process. The comparison among the measurements assured accurate quality control during the whole process of conductor production. In addition, $I_{c}$ measurements in fields up to $6 \mathrm{~T}$ on the complete CMS conductors using the MaRiSA test facility are reported.
\end{abstract}

Index Terms-Aluminum stabilized super-conductors, CMS solenoid, NbTi, strand properties.

\section{INTRODUCTION}

$\mathbf{T}$ HE CMS experiment (Compact Muon Solenoid) is a general-purpose proton-proton detector designed to run at the highest luminosity at the LHC. Distinctive features of the CMS detector include a high magnetic field solenoid coupled with a multilayer muon system, a fully active scintillating crystal electromagnetic calorimeter, a tile hadronic calorimeter, and a pow-

Manuscript received October 20, 2003.

B. Blau was with the Swiss Federal Institute of Technology Zurich (ETH), CH-8093 Zürich, Switzerland. He is now with the Paul Scherrer Institute (PSI), 5232 Villigen PSI, Switzerland (e-mail: bertrand.blau@psi.ch).

D. Campi and B. Curé are with CERN, 1211 Geneva 23, Switzerland.

M. Greco is with INFN-Genova, 16146 Genova, Italy.

F. Kircher and L. Vieillard are with CEA Saclay, Dapnia/STCM, 91191, Gif-sur-Yvette, France.

R. Liikamaa and J. Seppälä are with Outokumpu Poricopper Oy, 28330 Pori, Finland.

R. Smith is with Fermi National Accelerator Laboratory, Batavia, IL 60510-0500 USA.

Digital Object Identifier 10.1109/TASC.2004.829716

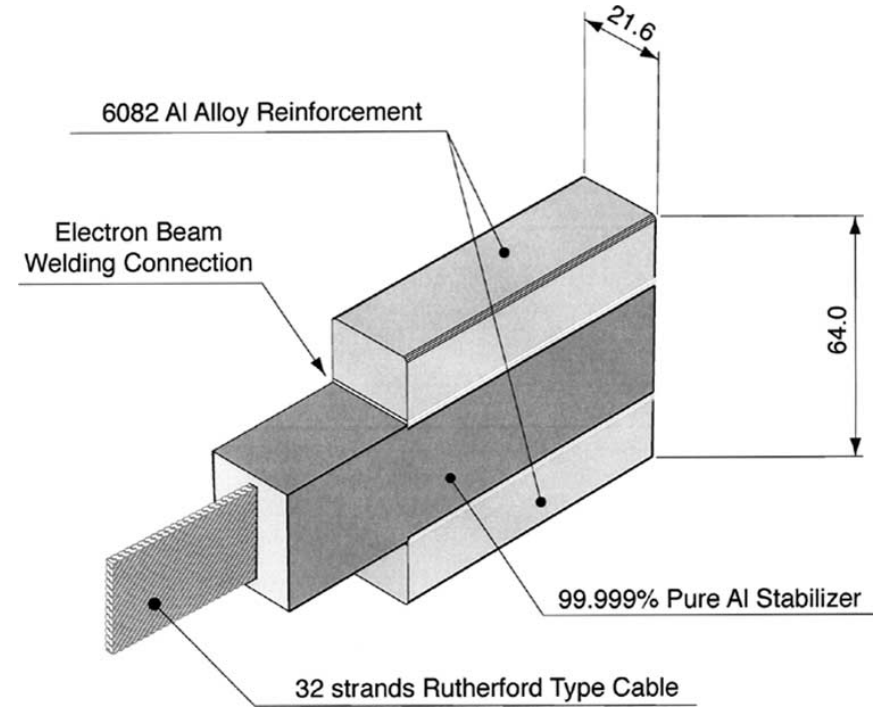

Fig. 1. The distinct feature of the CMS conductor is its sandwich-like structure. The high purity aluminum stabilized NbTi conductor is mechanically reinforced by two high yield strength AA sections.

erful inner tracking system. The design field of the superconducting CMS solenoid is $4 \mathrm{~T}$, the magnetic length is $12.5 \mathrm{~m}$ and the free diameter of the bore is $6 \mathrm{~m} \mathrm{[1].}$

As reported previously in detail, e.g., in [2], the main peculiarity of the CMS magnet among other existing thin detector solenoids is its sandwich-type aluminum-stabilized superconductor. This special feature was chosen in order to have a mechanically self-supporting winding structure. The coil is made of 20 conductors each having a length of $2550 \mathrm{~m}$. The CMS conductor consists of $32 \mathrm{NbTi} / \mathrm{Cu}$ strands wound into a Rutherford type cable. The cable is embedded in a high purity aluminum sheath for electrical and thermal stabilization by using a special adopted co-extrusion technology [2]. This rectangular assembly is called the insert. Since the yield strength of high purity aluminum is not sufficient to counteract the electromagnetic forces of the coil, the conductor is reinforced by two aluminum alloy (EN AW-6082) sections as shown in Fig. 1. The yield strength of this material at $4.2 \mathrm{~K}$ is $\mathrm{R}_{\mathrm{p} 0.2}=428 \mathrm{MPa}$ (in final T6 temper) [3]. The aluminum alloy sections are joined by continuous electron beam welding to the insert, using a specially designed production line [4].

Nineteen lengths of CMS conductor have been successfully finished to date. Completion of conductor production is expected for November 2003. The production time of the conductor was about three years starting with the cabling of the first Rutherford cable and ending with the welding of the last unit length. 
TABLE I

CMS STRAND CHARACTERISTICS

\begin{tabular}{lc}
\hline \hline \multicolumn{1}{c}{ Parameter } & Quantity \\
\hline Superconducting material & $\mathrm{Nb} 47 \pm 1 \mathrm{Wt} \% \mathrm{Ti}$ \\
Strand diameter & $1.280 \pm 0.005 \mathrm{~mm}$ \\
$(\mathrm{Cu}+$ Barrier $): \mathrm{NbTi}$ & $1.1 \pm 0.1$ \\
$\mathrm{Nb}:(\mathrm{Nb}+\mathrm{NbTi})$ & 0.021 \\
Filament diameter & $<40 \mu \mathrm{m}$ \\
Number of filaments & $\geq 552$ \\
Twist pitch & $45 \pm 5 \mathrm{~mm}(\mathrm{Z})$ \\
$\mathrm{Cu}$ RRR & $>100$ \\
$I_{c}$ at $5 \mathrm{~T}, 4.2 \mathrm{~K}, 10 \mu \mathrm{V} / \mathrm{m}$ & $>1925 \mathrm{~A}$ \\
$\mathrm{n}-\mathrm{value}$ of resistive transition at $5 \mathrm{~T}$ & $>40$ \\
Strand unit length & $2750 \mathrm{~m}$ \\
\hline \hline
\end{tabular}

About $1950 \mathrm{~km}$ of superconducting strands were used to produce the required number of conductors. This paper discusses the statistics of various strand parameters, which were monitored over the whole production. Since the strands are subjected to potentially dangerous high temperatures and mechanical strain during the different conductor production steps it was important to trace the critical current properties of the strand as it progressed through the conductor fabrication process. The comparison among the measurements assured accurate quality control during the whole process of conductor production.

\section{THE SUPERCONDUCTING STRAND}

The superconducting strand is a multifilamentary wire with a diameter of $1.28 \mathrm{~mm}$ composed of 552 high homogeneity $\mathrm{NbTi}$ filaments sheathed with a $\mathrm{Nb}$ barrier and embedded in a high purity copper matrix. The required minimum critical current is $1925 \mathrm{~A}$ at $5 \mathrm{~T}$ and $4.2 \mathrm{~K}$, corresponding to a critical current density of $3074 \mathrm{Amm}^{-2}$ in the superconductor, which is at the upper limit of present industrial capability. The strands are heat treated at the final stage to achieve a copper RRR (residual resistivity ratio) above 100 . The main required parameters of the strand are summarized in Table I. All 708 contractual unit lengths, i.e., $1947 \mathrm{~km}$ (corresponding to $18650 \mathrm{~kg}$ ), were produced by Outokumpu Poricopper Oy and delivered on 158 spools. The material was sufficient to produce 22 cables. The strand produced from one billet had a length of typically $14000 \mathrm{~m}$.

Intensive quality control was performed on the CMS strands during manufacturing, including continuous eddy current checks and diameter measurements after final drawing. On test samples taken from both ends of each finished strand the critical current, the n-value, the $(\mathrm{Cu}+$ barrier $) / \mathrm{NbTi}$ ratio and the copper RRR were measured. In contrast to a former publication [5] the statistics of various strand parameters presented here comprise the measured data of all 158 delivered spools required for the CMS solenoid.

\section{A. Critical Current}

$I_{c}$ was measured at $4,5,6$, and $7 \mathrm{~T}$ at $4.2 \mathrm{~K}$ using a $10 \mu \mathrm{V} / \mathrm{m}$ criterion. In Fig. 2 the $I_{c}$ values at $5 \mathrm{~T}$ of all delivered lengths are presented. All samples exceeded the specification. The average

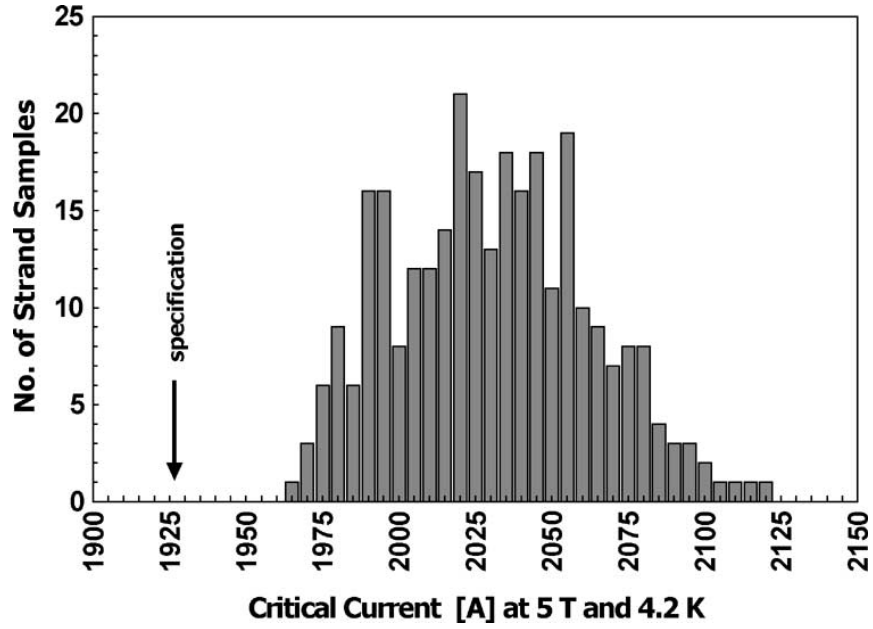

Fig. 2. Critical currents at $5 \mathrm{~T}$ and $4.2 \mathrm{~K}$ of strand samples from all delivered lengths.

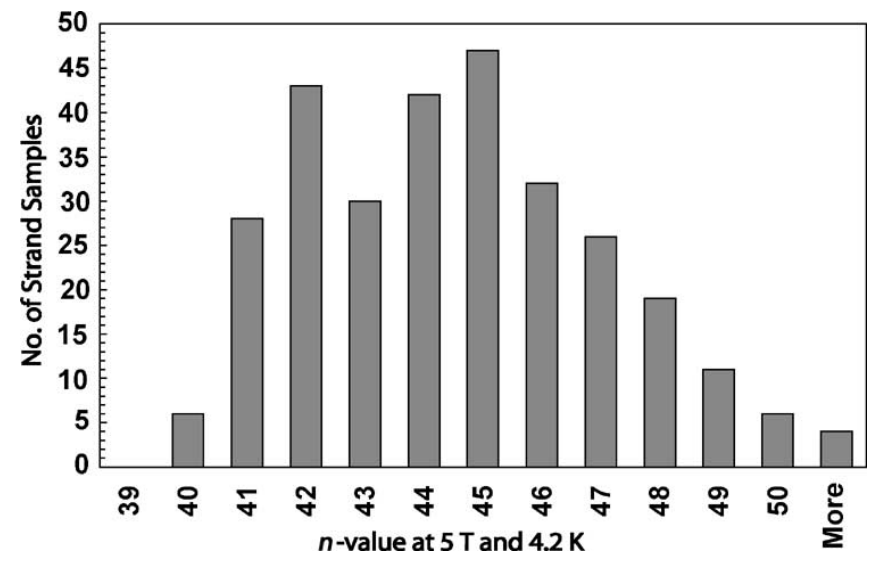

Fig. 3. Statistics on the n-values (at $5 \mathrm{~T}$ ) of all CMS strand samples.

critical current is $2029 \pm 32$ A corresponding to a critical current density of $3240 \pm 51 \mathrm{~A} / \mathrm{mm}^{2}$.

\section{B. $n$-Value}

The $n$-value which characterizes the transition from the superconducting to the resistive state was derived from $I_{c}$ measurements at $5 \mathrm{~T}$. It was calculated by using the equation

$$
\mathrm{n}=\left(\log \left(\frac{I_{c, 100 \mu \mathrm{V} / \mathrm{m}}}{I_{c, 10 \mu \mathrm{V} / \mathrm{m}}}\right)\right)^{-1}
$$

The higher the $n$-value, the sharper the transition and the better the wire quality. The minimum required value for the CMS strand was 40.

The statistics of all measured samples is shown in Fig. 3. The average value of all 294 strand samples was found to be $44.6 \pm$ 2.6. None of the samples had a value below 40 .

\section{The $(\mathrm{Cu}+$ barrier $) /$ NbTi Ratio}

The parameter which describes the ratio between the amount of normal conducting to superconducting material is the $\mathrm{Cu}+$ barrier)/NbTi ratio. We regard the pure Niobium barrier material here also as nonsuperconductive material since Niobium becomes normal conducting at the magnetic field levels this 


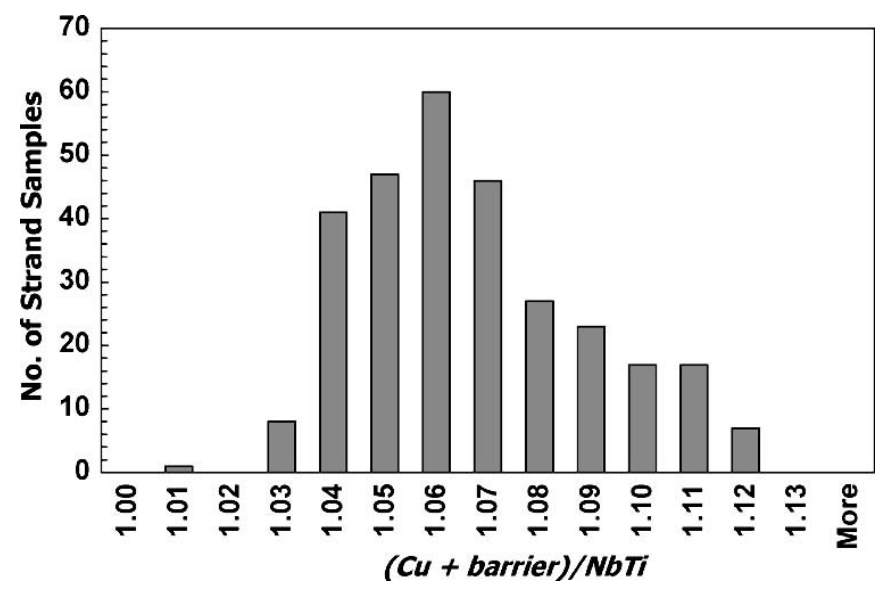

Fig. 4. Statistics on the $(\mathrm{Cu}+$ barrier $)$ /NbTi ratio of all CMS strand samples.

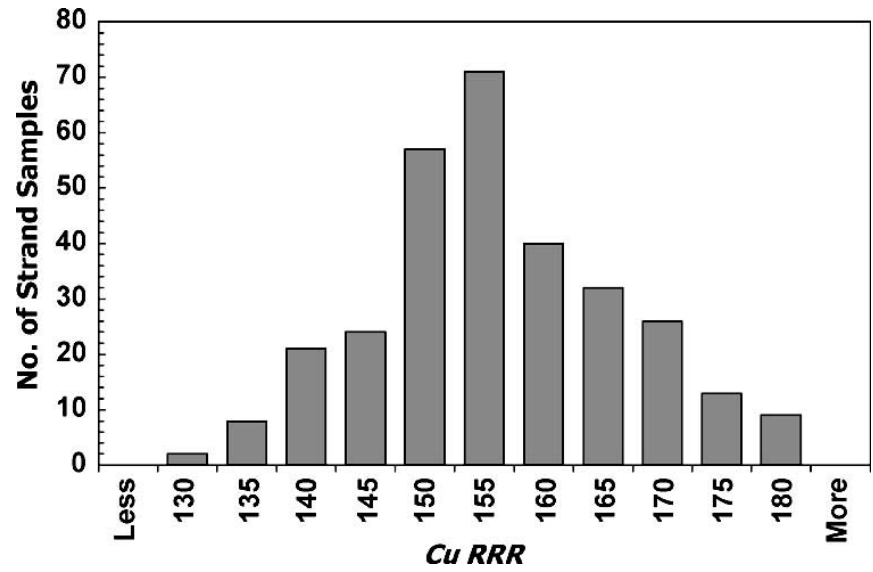

Fig. 5. Statistics on the strand copper RRR of all CMS strand samples.

conductor will be applied to. This ratio is experimentally determined on short samples by etching away the copper matrix and weighing the remaining NbTi filaments. The statistics for all 294 strand samples is shown in Fig. 4. On average a ratio of $1.06 \pm 0.02$ was found. All strands meet the specification, which is $1.1 \pm 0.1$.

\section{Strand Copper RRR}

The residual resistivity ratio RRR of the copper matrix of the strands is required to exceed 100 , both for electrical and thermal stability of the strand and for sufficient ductility, which is important for the cable manufacturing process. A final annealing heat treatment is performed on the strands during production leading to a good level of uniformity among the different strands as can be seen in Fig. 5, which shows the statistics of all 294 samples. The mean value of the copper RRR was found to be $156 \pm 11$.

\section{COMPARISON OF STRAND $I_{c}$ PROPERTIES DURING DIFFERENT STEPS OF CONDUCTOR FABRICATION}

Due to the special design of the CMS conductor the superconducting strands are subjected to potentially dangerous high temperatures during extrusion and electron beam welding as well as to large mechanical strain during cabling. In order to assure good quality control it is therefore necessary to monitor the current carrying capabilities during different production

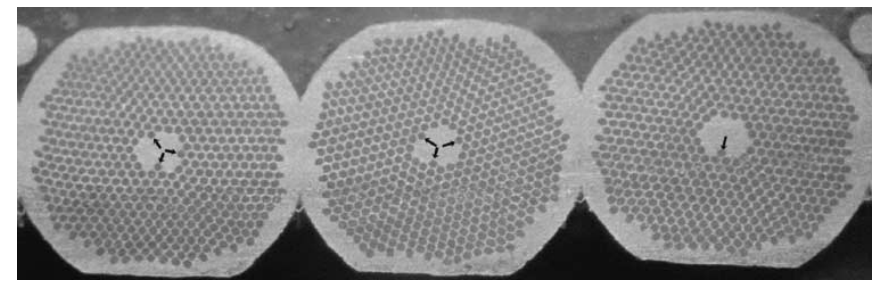

Fig. 6. Enlarged photograph of a CMS cable section displaying three strands with different configuration of filaments in the core region for identification purpose (indicated by arrows)

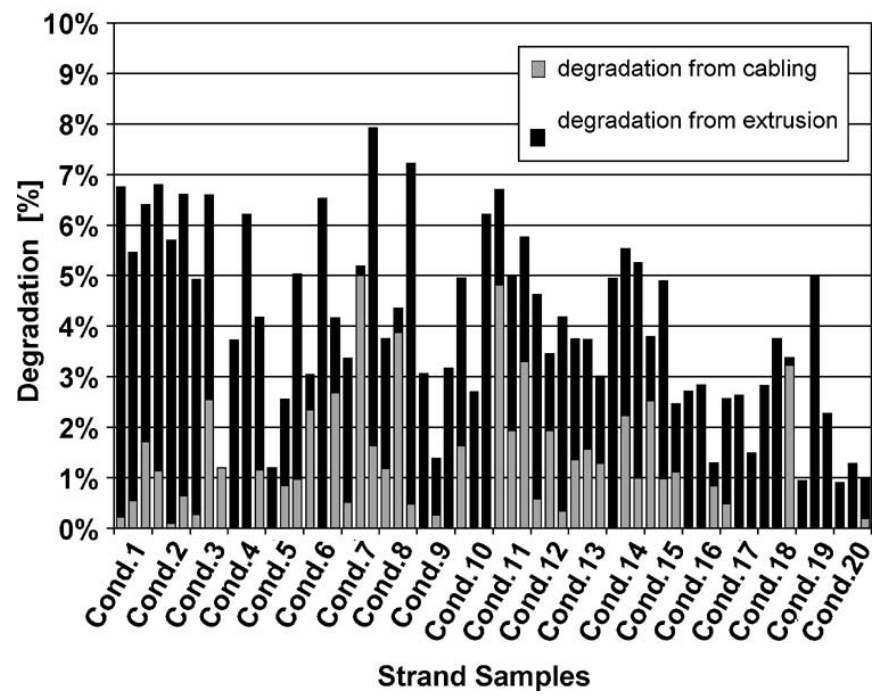

Fig. 7. Statistics on the $I_{c}$ degradation on extracted strands from the CMS conductor due to the cabling (gray) and the extrusion (black) process.

after cabling and extrusion and typically three strands were extracted from each sample to determine the critical current. The key point is that always the same strands were extracted from the different samples of one conductor in order to have a direct comparison of the $I_{c}$ properties of each single strand. To trace individual strands within the 32-strand Rutherford cable, eight distinctive patterns of filament arrangements were selected during stacking of monofilaments. Each cable is made with an individually defined cabling pattern. The cabling pattern is recognizable with the help of a microscopic analysis of the sample cross section. With this information it is possible to extract always the same specific strands from all samples of one conductor production. Fig. 6 shows a microscopic photograph of a section of the Rutherford cable where three strands with distinct filament pattern in the boundary region between filamentary zone and the pure copper core are visible.

A rather low compaction ratio (87\%) has been chosen for the CMS cable in order to ensure small critical current degradation due to strain and filament damage. In Fig. 7 the $I_{c}$ degradation due to cabling (gray columns) is presented for three strands of each of the twenty conductors to be used in the CMS magnet. All $I_{c}$ measurements were performed at CEA Saclay. The degradation, which was found, varies between 0 and $5.0 \%$, the average being $1.0 \%$. The same plot also displays the additional degradation due to the extrusion process. Since the strands were subjected to extrusion temperatures as high as $400^{\circ} \mathrm{C}$ for several seconds some degradation was expected. On 59 strands coming 


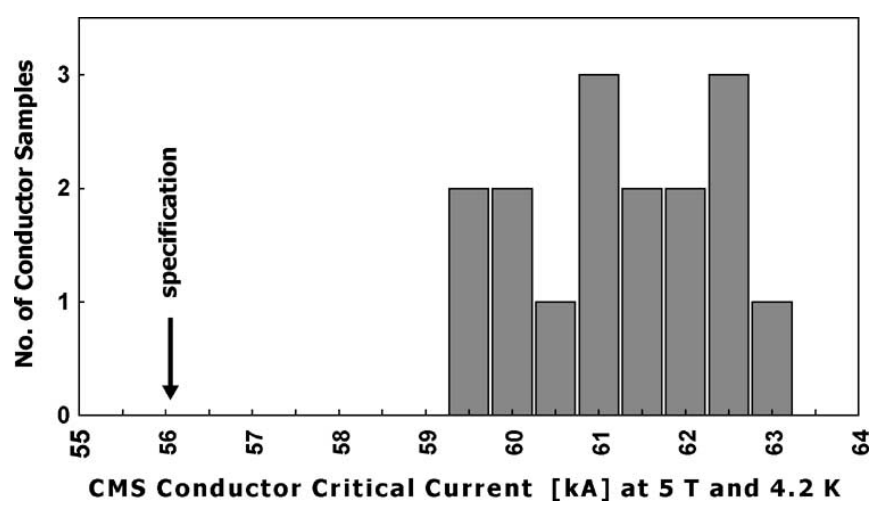

Fig. 8. Statistics on the critical current of 16 finished CMS conductors.

from all twenty conductors degradation due to extrusion between 0.2 and $6.7 \%$ was found with an average of $3.1 \%$. Based on these data a mean total degradation of $4.1 \pm 1.8 \%$ can be evaluated. The average $I_{c}$ value (at $5 \mathrm{~T}, 4.2 \mathrm{~K}$ ) of the 60 virgin strands considered here is 2029 A, i.e., $5 \%$ above the specified value of $1925 \mathrm{~A}$. The average $I_{c}$ value which was found on the extracted strands from the extruded conductor is 1947 A, i.e., even after cabling and extrusion the average strand critical current is still $1 \%$ above the specified virgin value.

\section{IV. $I_{c}$ MEASUREMENTS ON THE COMPLETE CMS CONDUCTOR}

The last step in the production of the CMS conductor is the electron beam welding of the two reinforcement bars to the insert. The main advantage of the electron beam welding process is that the energy deposition during welding is localized to a narrow melted zone. This is particularly important for avoiding severe critical current degradation of the superconducting cable located only $4.7 \mathrm{~mm}$ distant from the welded interface. For final quality control the critical current of the complete CMS conductor is measured by using the MaRiSA test facility at INFN-Genova [6]. These measurements are the supreme tests because they yield the current carrying capabilities of the conductor under most similar conditions to the operation in the magnet.

Fig. 8 shows the statistics on the critical current at $5 \mathrm{~T}$ and $4.2 \mathrm{~K}$ of the $16 \mathrm{CMS}$ conductors measured to date. The mean critical current is $61.0 \pm 1.0 \mathrm{kA}$. The accuracy of the MaRiSA facility is about $\pm 2 \%$. These results show that all values are at least $6 \%$ above the specified value of $56 \mathrm{kA}$ for the finished conductor. For comparison, the nominal current of the CMS coil at $4.2 \mathrm{~K}$ is $19.5 \mathrm{kA}$ creating a field of $4.6 \mathrm{~T}$ at maximum on the conductor.

In order to evaluate the additional degradation due to electron beam welding we divide the measured conductor current by 32 to get the average strand $I_{c}$. This can be compared with the mean value of the measured strand $I_{c}$ 's extracted from samples of the corresponding insert. Fig. 9 shows the additional degradation derived by this method for all sixteen conductors measured to date. Due to the fact that both the strand $I_{c}$ values of the insert values have $2 \%$ uncertainty the error bars are rather large in some cases.

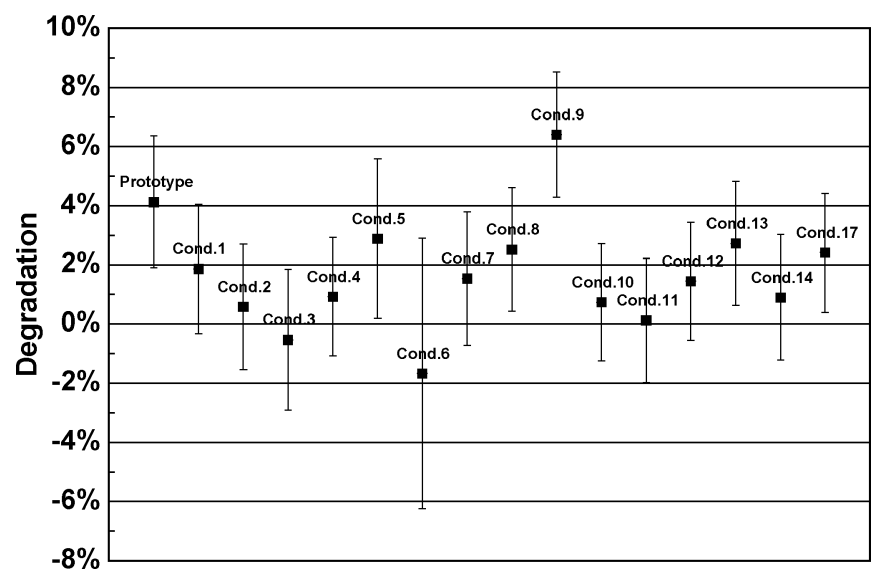

Fig. 9. Additional degradation of critical current of the CMS conductor due to electron beam welding.

It can be seen from the plot that the additional degradation due to electron beam welding is very small, if there is any. Purely arithmetically, we find an additional mean degradation of $1.7 \%$. In the worst case it is $6.4 \%$.

\section{CONCLUSIONS}

The production of the CMS conductor will be finished in November 2003. Because of the unconventional design of the CMS conductor it was of great importance to trace carefully the critical current properties of the superconducting strands as they progressed through the conductor fabrication process. A careful set-up of the various steps of the fabrication process assured that the total $I_{c}$ degradation does not exceed $9 \%$ with respect to the critical current of the virgin strands. In detail we observed degradation of $5.0 \%$ at maximum due to cabling, of $6.7 \%$ in worst case due to extrusion and of $6.4 \%$ at maximum due to electron beam welding. The actually measured average critical current of the virgin strand is $2029 \mathrm{~A}$ at $5 \mathrm{~T}$ and $4.2 \mathrm{~K}$ instead of $1925 \mathrm{~A}$, which was specified, i.e., 5\% above the specification. Due to the rather challenging conductor fabrication process a total degradation of $9 \%$ with respect to the design value of the strand $I_{c}$ was allowed, i.e., a conductor critical current of $91 \% \cdot 32 \cdot 1925 \mathrm{~A}=56 \mathrm{kA}$ was specified. The actually measured critical current we found was $59.4 \mathrm{kA}$ or higher, thus at least $6 \%$ above specification.

\section{REFERENCES}

[1] A. Herve et al., "Status of the Construction of the CMS Superconducting Magnet at LHC," .

[2] B. Blau et al., "The CMS conductor," IEEE Trans. Appl. Supercond., vol. 12, no. 1, pp. 345-348, March 2002.

[3] B. Curé et al., Mechanical Properties of the CMS Conductor, , this conference.

[4] R. Folch et al., "Continuous EB welding of the reinforcement of the CMS conductor," IEEE Trans. Appl. Supercond., vol. 12, no. 1, pp. 372-375, March 2002.

[5] B. Curé et al., "The superconducting strand for the CMS solenoid conductor," IEEE Trans. Appl. Supercond., vol. 12, no. 1, pp. 1014-1017, March 2002.

[6] M. Greco, P. Fabbricatore, and R. Musenich, "Critical current measurements on the CMS stabilized conductor," IEEE Trans. Appl. Supercond., vol. 12, no. 1, pp. 381-384, March 2002. 